\title{
The Pollution Of Bromate In Drinking Water And Its Detection Methods
}

\author{
Yamei Han ${ }^{1, a}$, Minmin Jiang ${ }^{2, b}$, Menglong Xing ${ }^{2, c}$, Haixiang Li ${ }^{\star, d}$ \\ ${ }^{1}$ College of Environmental Science and Engineering, GuiLin University of Technology, Guilin \\ 541006, China \\ ${ }^{2}$ College of Environmental Science and Engineering, GuiLin University of Technology, Guilin \\ 541006, China \\ "Guangxi Key Laboratory of Environmental Pollution Control Theory and Technology, Guilin \\ University of Technology, Guilin 541004, China \\ a2537585267@qq.com,b505586101@qq.com, ${ }^{\mathrm{c} 1520051565 @ q q . c o m,{ }^{\mathrm{d}} 120798590 @ q q . c o m}$
}

Keywords: Drinking water; Bromate; Ion chromatography; Detection method

Abstract. Bromate $\left(\mathrm{BrO}_{3}{ }^{-}\right)$is an oxidized contaminant produced from bromide $\left(\mathrm{Br}^{-}\right)$during ozonation and advanced oxidation of drinking water. which is considered to be a potential 2B-level carcinogen, would couse serious harm to human health. This paper reviewed the formation process of bromate, the main influence factors, detecting bromate methods, in particular ion chromatography and pre-treatment methods were described in detail. And this paper pointed out that a wider range of applications of ion chromatography detection in drinking water.

\section{Introduction}

With the improvement of people's living standards, they have the higher requirement of the quality of drinking water; the same time, the rapid development of social industrialization causes of water pollution problems have become more and more serious, with traditional conventional water treatment technology has been unable to meet the "drinking water health standards" (GB5749-2006).however,ozone is widely used in drinking water treatment technology due to its highly efficient strong oxidizing and disinfection [1-3]. But, when the ozone oxidation of bromine-containing water will generate byproducts bromate. in 1992, the World Health Organization (WHO) published the safe use of the examination report confirmed bromate is an oxidation carcinogens that can cause kidney and bladder tissues of animals occurrence cancerous [4, 5]. Bromate has been IRAC (International Agency for Research on Caner, IRAC) as 2B-level potential carcinogen(higher carcinogenic potential), the US EPA studies have shown that a normal adult drinking bromate concentration of $5.0 \mu \mathrm{g} / \mathrm{L}$ in drinking water, the lifetime carcinogenicity rate is $10^{-4}[6,7]$. Therefore, many countries made the maximum allowable concentration of bromate clear in drinking water quality standards.In 2004, World Health Organization (World Health Organization, WHO) "Guidelines for Drinking Water Quality" revised bromate limit from 25 $\mu \mathrm{g} / \mathrm{L}$ to $10 \mu \mathrm{g} / \mathrm{L}$, China's "drinking water health standards" (GB5749-2006) also set the bromate concentration in drinking water lower than $10 \mu \mathrm{g} / \mathrm{L}[2,8]$. As disinfection byproducts, bromate levels in drinking water is very low, usually be measured by $\mu \mathrm{g} / \mathrm{L}$ magnitude [9]. Therefore, how to highly efficient detect the trace amounts of bromate and the content of bromate in drinking water is very important. 


\section{The formation process of bromate and the main affecting factors}

Numerous studies showed that ozone treatment of drinking water during the bromine ions with ozone and hydroxyl radical $(\bullet \mathrm{OH})$ joint reaction of bromate, in this process, including six oxidation states of bromine is shown in Table 1 [10]. Produce bromate byproduct contains three main ways [11]: Direct individually $\mathrm{O}_{3}$ molecule oxidation; $\mathrm{O}_{3}$ molecules are oxidized after $\bullet \mathrm{OH}$ oxidation; $\bullet$ $\mathrm{OH}$ is oxidized after $\mathrm{O}_{3}$ molecule oxidation. The oxidation process is shown in Fig.1.Generally it believed that ozone in two stages of oxidation [3]: the initial oxidation stage due to the action of $\bullet \mathrm{OH}$, bromate levels increased rapidly; the second stage ozone oxidation is due to $\bullet \mathrm{OH}$ and $\mathrm{O}_{3}$ molecules together, bromate increase more slowly. Ozekin et al. [12] showed that $\bullet \mathrm{OH}$ plays a dominant role during the formation of bromate.

Table 1 Bromine species formed during bromate formation oxidation states and important oxidants

\begin{tabular}{|c|c|c|}
\hline Chemical formula & Valence of bromine & Substances controling of oxidize \\
\hline $\mathrm{Br}^{-}$ & $-\mathrm{I}$ & $\mathrm{O}_{3}, \cdot \mathrm{OH}$ \\
\hline $\mathrm{Br} \bullet$ & 0 & $\mathrm{O}_{3}$ \\
\hline $\mathrm{HOBr}$ & $+\mathrm{I}$ & $\bullet \mathrm{OH}$ \\
\hline $\mathrm{OBr}^{-}$ & $+\mathrm{I}$ & $\mathrm{O}_{3}, \cdot \mathrm{OH}_{2}, \mathrm{CO}_{3}{ }^{2-}$ \\
\hline $\mathrm{OBr}^{\bullet}$ & $+\mathrm{II}$ & - \\
\hline $\mathrm{BrO}_{2}^{-}$ & $+\mathrm{III}$ & $\mathrm{O}_{3}$ \\
\hline $\mathrm{BrO}_{3}^{-}$ & $+\mathrm{V}$ & - \\
\hline
\end{tabular}

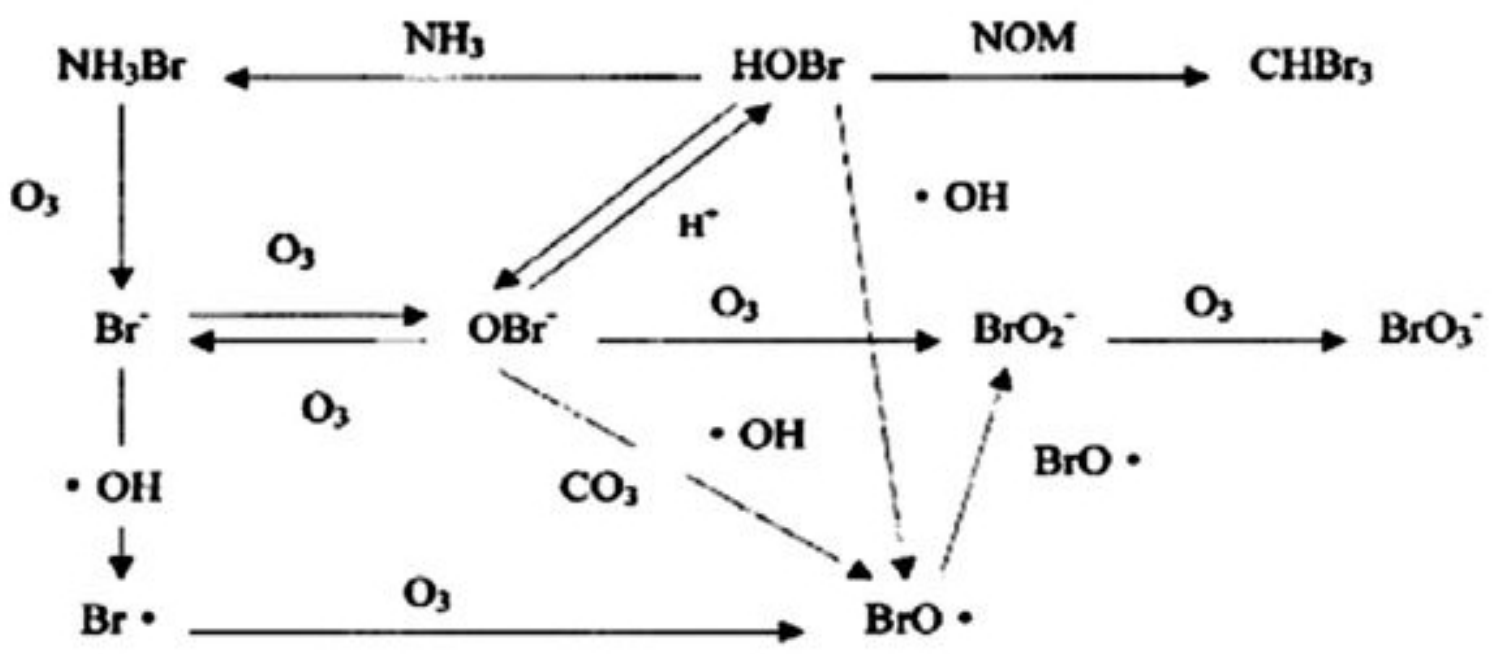

Fig.1 Reaction scheme for bromate formation

The main factors bromate formation include ozone dosage, $\mathrm{Br}^{-}$concentration, $\mathrm{pH}$, temperature, alkalinity and the like. Siddiqui and Army et al $[13,14]$ pointed out that the process of ozone oxidation of bromate, $\mathrm{O}_{3}$ and $\mathrm{Br}^{-}$there exist a critical concentration, when the concentration is lower than this, less bromate generation amount; when above the critical concentration, the higher $\mathrm{O}_{3}$ and $\mathrm{Br}^{-}$concentration, the higher the amount of bromate is generated, until the ozone is completely consumed. Numerous studies showed that increasing the $\mathrm{pH}$ of the water or rising the water temperature would promote the generation of bromate. Krasner et al [15] found the generation of bromate increases with the rising water alkalinity as the $\mathrm{pH}=8$. While $\mathrm{pH}=7$ the formation of bromate decreased with increasing water alkalinity. 


\section{The detection method of bromate}

The traditional methods of detecting bromate include chemical titration, spectrophotometry [16-18] and gas chromatography [19]. However, bromate content in drinking water is very low, in order to reduce the impact of high levels of other common anions its detection requires prior removal of high levels of other anions, and traditional chemical titration and spectrophotometric methods of pre-treatment process is relatively cumbersome, they take a long time; even more critical is that these conventional chemical analysis methods generally have low sensitivity, can not meet the bromate testing requirements in drinking water and other shortcomings. Therefore, the conventional chemical analysis methods are generally content of flour and flour products for the determination of bromate. Although gas chromatography meet the drinking water of trace bromate detected, but because this method requires a derivatization reaction, and the operating derivatized reaction is difficult to control, and is derived reagents toxic, less secure also limits the use of gas chromatography in the determination of bromate in drinking water. At present, the commonly used measurement of bromate is ion chromatography method and some of its new combined techniques.

Ion chromatography. Ion chromatography is based on the principle of mutual exchange between the ions, while the mixed anion and cation separation, and the separated ions are analyzed qualitative and quantitative detection. In August 1993, the US Environmental Protection Agency (EPA) for the first time set ion chromatography as a standard method for determining bromate in water [20]. Ion chromatography generally consists of infusion pumps, injection valve, separation column, inhibition column and detector of several parts. As other detection methods, the analysis by ion chromatography also requires the necessary pre-treatment.

Sample pre-treatment technology. The commonly used pre-treatment techniques of bromate detection include membrane treatment, microwave concentration method and eliminating interference of chlorine ions. Membrane treatment method usually use $0.22 \mu \mathrm{m}$ polysulfone or nylon membrane as the membrane.under mechanical pressure component to be tested all through the filter to remove particulate contaminants. The method is simple, low cost, rapid processing, and can effectively prevent contamination of the column, it is more commonly used sample pretreatment methods. But most filter can not tolerate the high pressure, so membrane treatment method can only be used off-line sample preparation.

Microwave method using concentrated thermal stability of bromate, concentrated by evaporation of the sample solution, so as to improve the detection limit of analysis. J Liu et al [21] used a concentrator column instead of loop connected to the six-way valve to achieve a large volume injection enrichment bromate, so that the injection volume up to $5 \mathrm{~mL}$, the concentration factor is about 240 times to the conventional injection and detection limit is $0.01 \mu \mathrm{g} / \mathrm{L}$. This method is relatively new sample preparation techniques, which have many advantages,such as fast, efficient, and improving the sensitivity of detection methods, etc.

In order to eliminate the interference of high concentration of chlorine to bromate ion analysis in drinking water, we need to reduce the concentration of chloride ions in drinking water, chlorine removal methods currently often used include the following three :(1) silver electrode electrolytic chlorine removal: through electrolytic silver, so that the chlorine generated by the electrolytic reaction of silver chloride, silver electrodes attached to the chlorine ions are removed. The method is simple, low cost, and the dechlorination effect is obvious. (2) precipitation of silver chloride method: adding silver nitrate in the sample make silver ions react with chlorine ions and generate precipitation of silver chloride, remove chloride ions in water. The method is simple, but not easy to control the amount of silver chloride reagent, the excess silver chloride ions would contaminate the column. (3) ions exchange method: the same principle is used of silver halide precipitation, ions 
exchange is connected to the styrene sulfonic acid silver ions into the ion exchange column when the sample solution includes chlorine, bromine and other halogen ion flows when the resin column, it will combine with the silver ions and graduate silver chloride or silver bromide precipitation, so as to eliminate chlorine, bromine plasma. This method has the quick and effective advantages, is currently the most widely used method of pretreatment.

The detection method of ion chromatography. After appropriate pretreatment, the sample solution is analyzed to detect by ion chromatography (IC). In recent years, the rapid development of ion chromatography instruments, not only produces a line eluent generator, detector types are also expanding. Currently, for bromate detector has three categories: (1) suppressed conductivity detection: bromate ions in water samples are separated by ion exchange column, it has been transformed into high conductivity weakly acidic bromate $\left(\mathrm{HBrO}_{3}\right)$ through suppressor, while the eluent is converted to low conductivity of weak electrolyte, so that the background conductivity is greatly reduced. The end result is significantly improved conductance test of bromate ions. Two common eluent system is carbonate-bicarbonate and hydroxide eluent systems. Carbonated water system has a large negative peak, which limit the large volume injection and further improve the sensitivity, and the system might be interfered by the air of carbon dioxide. While the hydroxide eluent be turned into water after go through suppressors, background conductivity is very low, the noise is small, and therefore it has the higher sensitivity, the lower detection limit. $\mathrm{H} \mathrm{Lu}$ and JZ Zhang [22] chosen $\mathrm{KOH}$ as eluent, using suppressed conductivity detection method made the detection limit of bromate less than $5 \mu \mathrm{g} / \mathrm{L}$. (2) post-column derivatization with UV detection: it is analytical methods that bromate reagent selectively react with derivatives, provide it with spectral absorption in the ultraviolet region. Commonly used agents are derived from bromide, iodide, dianisidine, magenta and the like. M Li et al. [23] used an ultraviolet detector to detecte UV absorption characteristics of four kinds of ions: iodate, iodide, bromate and bromide ions, comparated with ion chromatography Conductivity detection, this method has low detection limit, better chromatography peak shape, and better sensitivity and accuracy to meet the water quality standards of testing requirements. However, post-column derivatization means more complex, cumbersome operation, derivative conditions are difficult to control, and some derived reagents are harmful to people, limiting its application and development. (3) mass spectrometry technique: mass spectrometry (MS), tandem mass spectrometry (MS-MS) and inductively coupled plasma mass spectrometry (ICP-MS) as detectors have the higher selectivity and sensitivity than the conductivity detector, they can provide low to ng / L detection limit. M Han [24], who experimentally established IC- ICP- MS method to measure $\mathrm{Br}^{-}$and $\mathrm{BrO}_{3}^{-}$in drinking water, results showed that this method has good accuracy, low detection limit, high sensitivity, analysis time short for the determination of $\mathrm{Br}^{-}$and $\mathrm{BrO}_{3}{ }^{-}$in drinking water. But this technology has a high cost of analysis because of expensive equipments, and its widely used is limited.

\section{Conclusions and prospect}

In summary, ion chromatography is the best method for detecting trace amounts of bromate in drinking water, which is an efficient, rapid, sensitive and so on. With the emergence of new instruments and testing methods, such as the linking of ion chromatography and mass spectrometry, and other various types of analytical instruments, ion chromatography to be more widely used, the sensitivity and accuracy of detection has greater increase, there will be a broader development prospects in drinking water quality testing. 


\section{Acknowledgements}

This work was financially supported by the National Natural Science Foundation of China (51408146) and Guangxi Natural Science Foundation (2016GXNSFAA380204).

\section{References}

[1] S Ghafari, M Hasan, MK Aroua. Bioresource technology. Vol. 10 (2008), p. 3965-3974.

[2] R.S.Liu, Y.Zhang. Environmental Science and Technology. Vol. 12 (2010), p. 66-70 (In Chinese).

[3] J.F.Lu, Y.Zhang, Y.Wang, et al. Water Treatment Technology. Vol. 11 (2010), p. 5-10 (In Chinese).

[4] Assessment OoEHH. Public Health Goal for Bromate in Drinking Water. [2015]

[5] Wolf DC, Crosby LM, George MH, et al. Toxicologic pathology. Vol. 6 (1998), p. 724-729.

[6] Cartier T, Baert A, Cabillic P-J, et al. Environnement risques \& sante. Vol. 4 (2012), p. 316-321.

[7] J.Y.Liu, S.F.Mou. Environmental Chemistry. Vol. 2 (2002), p. 203-204 (In Chinese).

[8] Health standards for drinking water (GB5749-2006). Ministry of Health of the People's Republic of China. (2006).

[9] J.Liu, Q.Q.He, LL Yang, et al. Chromatography. Vol. 10 (2015), p. 1110-1114 (In Chinese).

[10] Von Gunten U. Water research. Vol. 7 (2003), p. 1469-1487.

[11] Von Gunten U, Oliveras Y. Environmental Science \& Technology. Vol. 1 (1998), p. 63-70.

[12] Ozekin K, Westerhoff P, Amy GL, et al. Journal Of Environmental Engineering-Asce. Vol. 5. (1998),p. 456-462.

[13] Siddiqui MS, Amy GL. Journal American Water Works Association. Vol. 1 (1993), p. 63-72.

[14] Siddiqui MS, Amy GL, Rice RG. Journal American Water Works Association. Vol. 10 (1995), p. 58-70.

[15] Krasner SW, Glaze WH, Weinberg HS, et al. Journal American Water Works Association. Vol. 1 (1993), p. 73-81.

[16]Q.G.Huo, L.Fan, N.N.Wu. Journal of Henan University of Technology: Natural Science Edition. Vol. 1 (2007), p. 10-12 (In Chinese).

[17]X.P.Li, R.X.Zhao, C.Y.Shen. Journal of Liaoning University of Petroleum \& Chemical Technology.Vol. 1 (2011),p. 9-12 (In Chinese).

[18]X.P.Liu, J.Chen, M.L.Jia. Chemical reagents.Vol. 2 (2008),p. 125-126 (In Chinese).

[19]P.Chen, S.S.Gu, Y.P.Lv, et al. Brand and Standardization.Vol. 2 (2010),p. 63 (In Chinese).

[20] Y.G.Li. Science \& Technology Review.Vol. 15 (2008),p. 77 (In Chinese).

[21]J.Liu, Q.Q.He, L.L.Yang, et al. Chromatography.Vol.10 (2015),p. 1110-1114 (In Chinese).

[22]H.Lu, J.Z.Zhang. Shandong Chemical Industry.Vol. 11 (2015),p. 77-8 (In Chinese).

[23] M.Li, H.Yu, X.R.Zeng. Chromatography.Vol. 3 (2014),p. 299-303 (In Chinese).

[24] M.Han, N.Jia, G.X.Zhao, et al. Journal of Anhui Agricultural Sciences.Vol. 21 (2015),p. 15-17

(In Chinese). 